\title{
Psychiatric Symptomatology in Medical Students in Lebanon: What about us?
}

Alfred Chabbouh, Carmen Al Haddad, Grace El Bejjani, Vanessa Daou

Supervised by Michele Chahoud, PhD

Affiliation: Lebanese University, Faculty of Medical Sciences

\section{Introduction}

Medical students are no strangers to mental health disorders, showing higher rates of psychiatric disorders compared to the general population (1). In addition to the inherent challenges of medical school, medical students in Lebanon are under increased stress due to the volatile sociopolitical situation in the country (2) which could increase the incidence of mental health disorders in this population. Therefore, the aim of this study is to screen for psychiatric symptoms in medical students in Lebanon and to determine the percentage of students that require further psychiatric evaluation. This study also highlights certain risk factors that are linked to a higher likelihood or more severe psychiatric symptoms in this population.

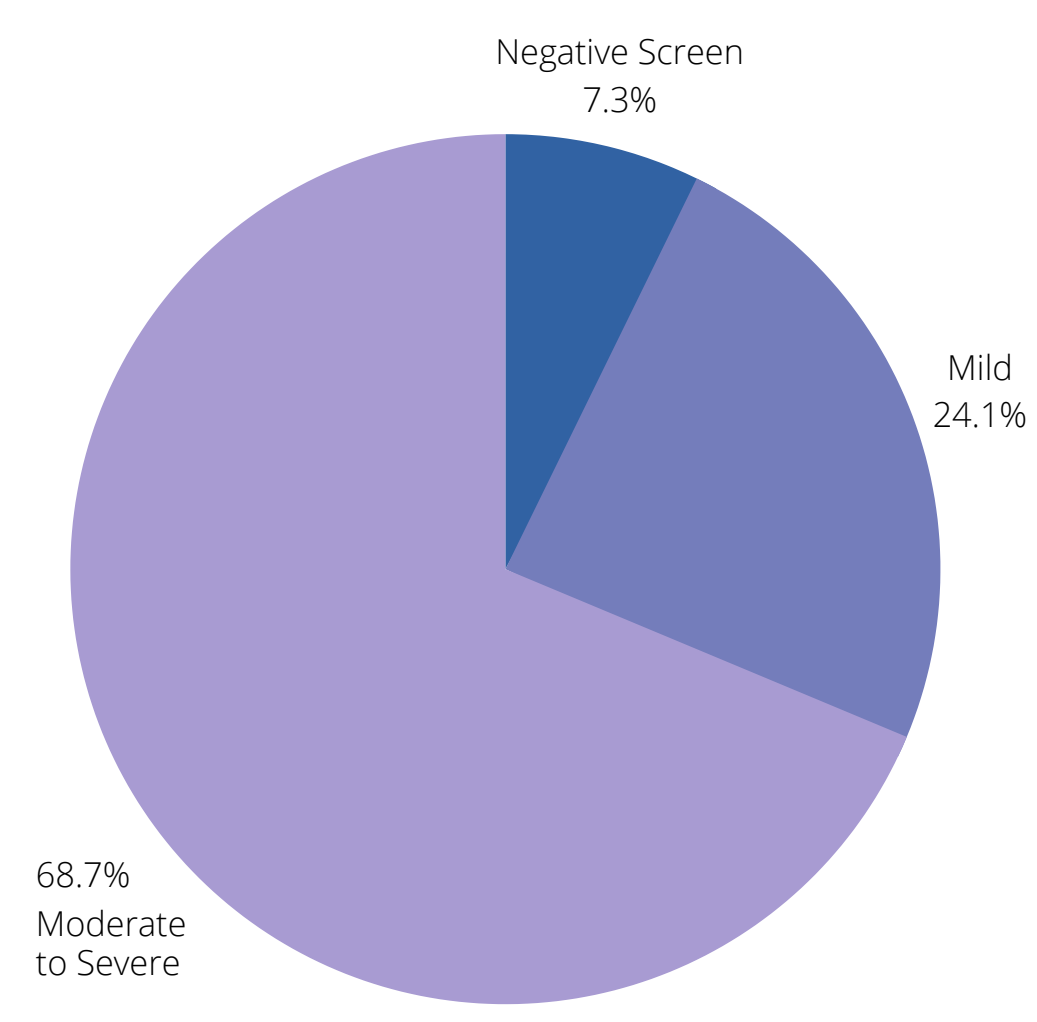
Fig 1. Mental health disorders symptoms
distribution based on APA's DXC Level 1

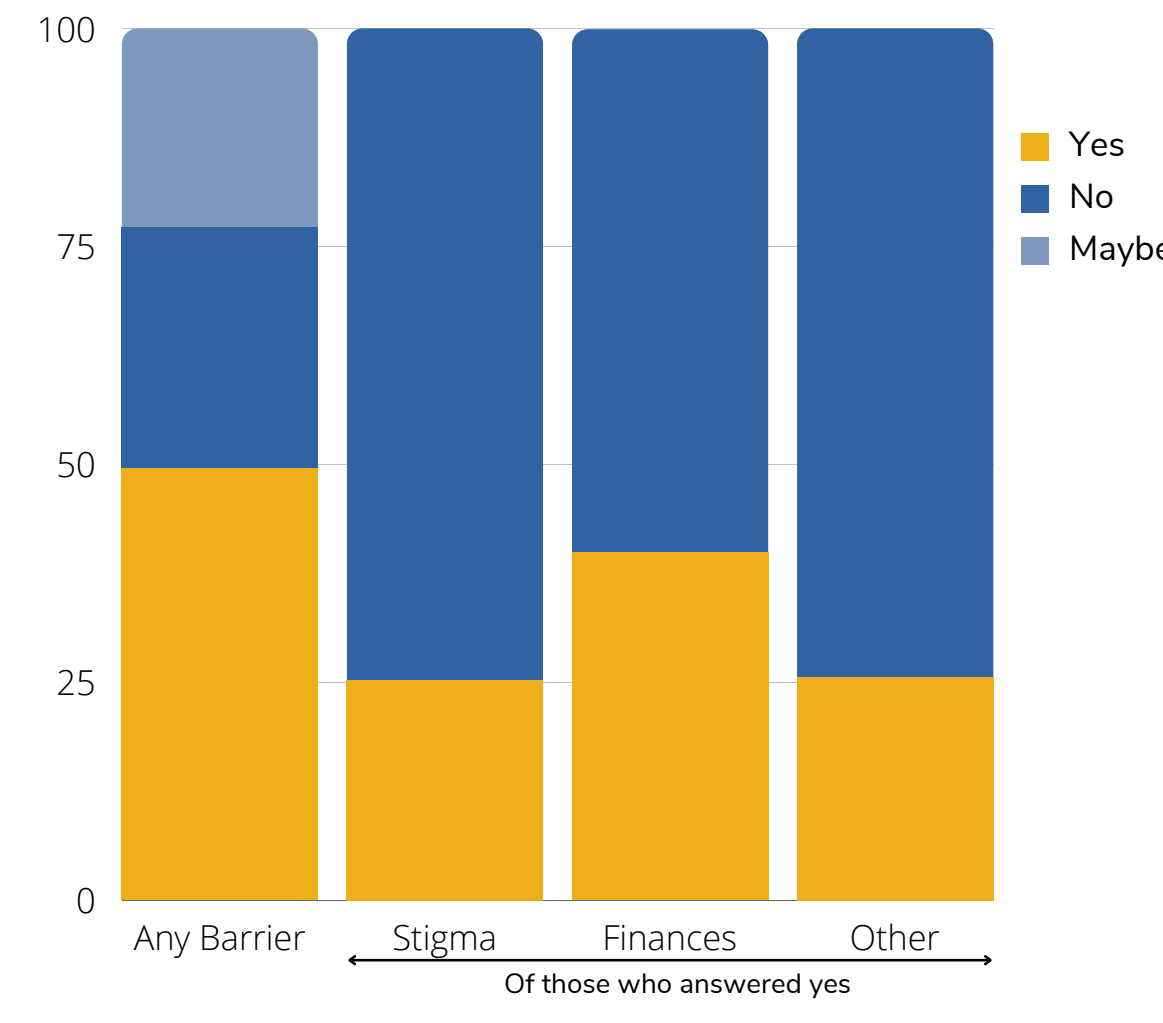

Fig 2. Barriers to seeking mental health services in medical students

\section{Methods}

An online questionnaire was sent asking about demographics, medical history, habits, and subjective experiences. The APA's "DSM-5 SelfRated Level 1 Cross-Cutting Symptom Measure - Adult" was used to screen for psychiatric symptoms in twelve different domains. A positive screen indicates the need for further psychiatric evaluation but not necessarily one or multiple psychiatric diagnoses.

\section{Results}

Responses were recorded from 364 medical students from all medical schools in Lebanon (more than $9 \%$ of the total number of medical students), of which $5.2 \%$ have already a diagnosed psychiatric disorder by a mental health professional. Of the non-diagnosed subpopulation (345 students), $92.75 \%$ showed a positive screen with $68.7 \%$ of the subpopulation reporting moderateto-severe symptoms. About half $(49.5 \%)$ of participants reported trouble seeking mental health services with finances being the major reason.

Experiencing past bullying was correlated to more likelihood and more severe reported symptoms. Students in private universities also reported more severe symptoms than their counterparts in the public university. Those who reported having strong social support were less likely to screen positively and ones who reported an increase in their experience of social pressure after entering medical school were more likely to screen positively. Finally, the suicide domain was significantly more positive in students who were previously bullied or LGBTQ+.

\section{Conclusion}

This study showcases the gravity of the situation in medical schools in Lebanon and the lack of proper care to students' wellbeing and affordable access to mental health services. Shouldn't the health of future healthcare providers be a priority?

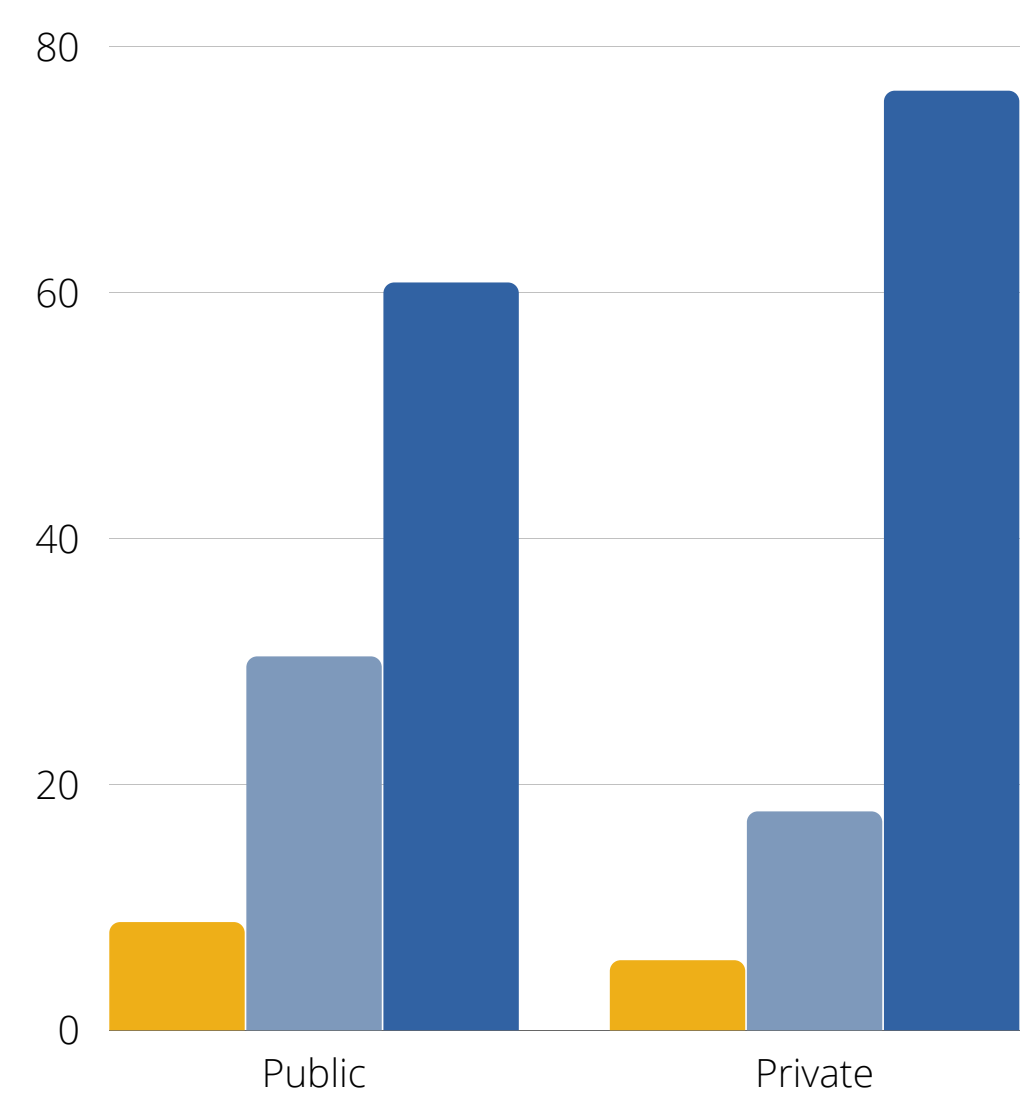

Fig 3. Difference in severity of mental health

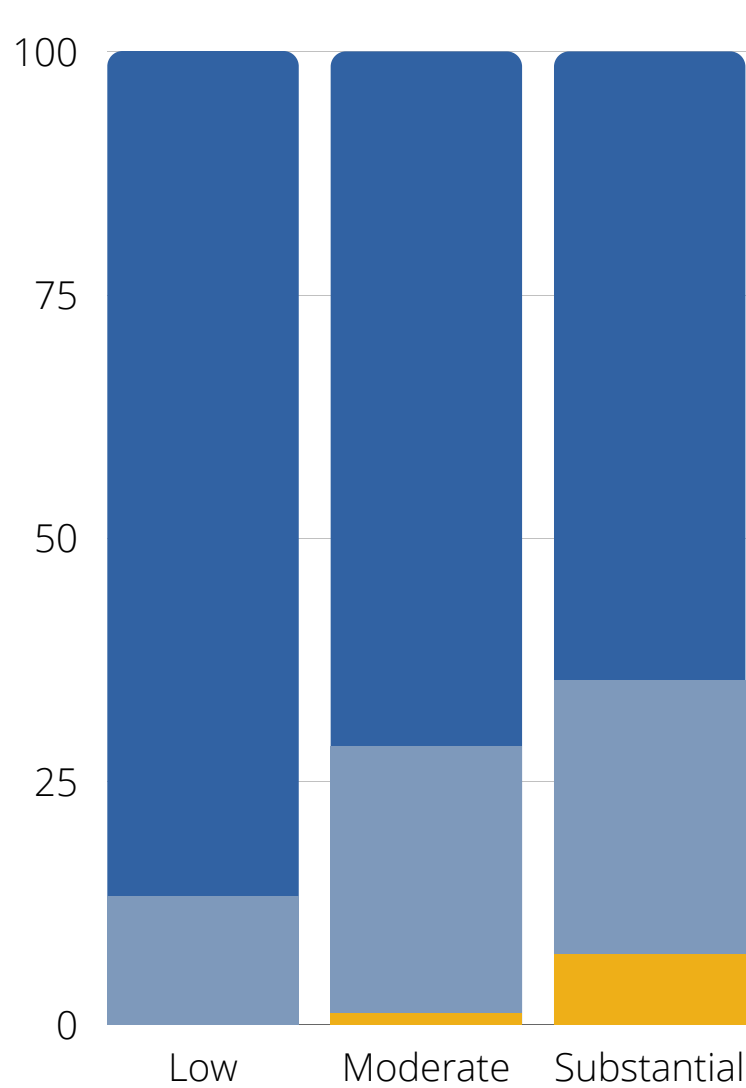

Fig 4. Mental health symptoms between social support levels

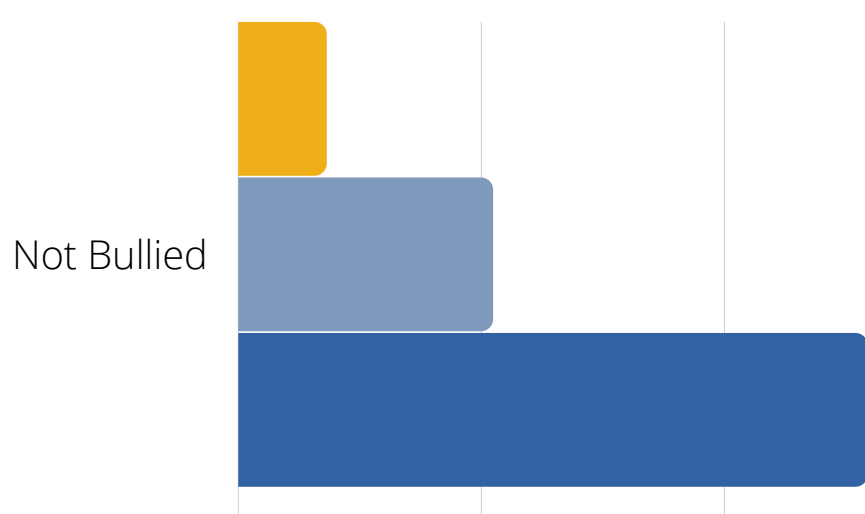
Moderate to
Severe Symptoms

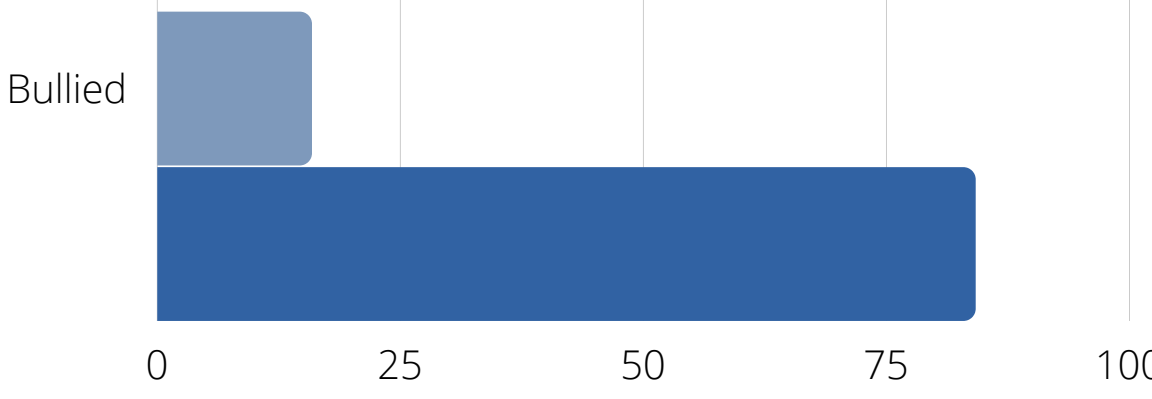

Fig 5. Severity of mental health symptoms depending on previous bullying experience 